\begin{tabular}{|c|l|}
\hline Title & A theorem on essential self-adjointness with application to Hamiltonians in nonrelativistic quantum field theory \\
\hline Author(s) & A rai, A sao \\
\hline Citation & Journal of Mathematical Physics, 32(8), 2082-2088 \\
\hline https://doi.org/10.1063/1.529178 \\
\hline Issue Date & 1991-08 \\
\hline Doc URL & http://hdl.handle.net/2115/13669 \\
\hline Rights & Copyright $\odot$ 1991 American Institute of Physics \\
\hline Type & article \\
\hline File Information & jmp32-82.pdf \\
\hline
\end{tabular}

Instructions for use 


\title{
A theorem on essential self-adjointness with application to Hamiltonians in nonrelativistic quantum field theory
}

\author{
Asao Arai \\ Department of Mathematics, Hokkaido University, Sapporo 060, Japan
}

(Received 9 October 1990; accepted for publication 26 February 1991)

\begin{abstract}
An abstract theorem is given on essential self-adjointness of operators in infinite direct sum of Hilbert spaces and is applied to a class of I Iamiltonians in nonrelativistic quantum field theory to prove their essential self-adjointness.
\end{abstract}

\section{INTRODUCTION}

In this paper we present an abstract theorem on essential self-adjointness of operators in infinite direct sum of Hilbert spaces and apply it to a class of Hamiltonians in nonrelativistic quantum field theory ( $Q F T$ ) to prove their essential selfadjointness.

About 20 years ago, D. Masson and W. K. McClary ${ }^{1}$ gave an interesting proof of the essential self-adjointness of the Hamiltonian of $\left(\phi^{4}\right)_{2}$ theory with a space cutoff. Their proof makes use of some specific properties of the interaction Hamiltonian acting in the boson Fock space over $L^{2}(\mathbb{R})$. We have found that their method can be formulated in an abstract way to give a criterion for essential self-adjointness of operators in infinite direct sum of Hilbert spaces. This is a background of the present work.

The outline of the present paper is as follows. In Sec. II we first state the abstract theorem mentioned above and then prove it. The proof of the theorem is quite similar to that of Masson and McClary in Ref. 1, but, we give it for completeness. Section III is devoted to the application of the theorem to a class of models in nonrelativistic QFT. Each model in the class describes a quantum system of a finite number of nonrelativistic particles interacting with some quantum scalar fields. In Sec. IV we discuss some examples: the linear polaron model, ${ }^{2-5}$ the RWA oscillator, ${ }^{6-8}$ a model of a bounded electron interacting with a quantized radiation field, ${ }^{9-11}$ their generalizations, and scalar quantum electrodynamics with cutoffs. ${ }^{12}$

\section{THE ABSTRACT THEOREM}

$$
\begin{aligned}
& \text { Let } \mathscr{H}_{n}, n=0,1,2, \ldots, \text { be Hilbert spaces and } \\
& \mathscr{H}=\underset{n=0}{\oplus} \mathscr{H}_{n}
\end{aligned}
$$

be the infinite direct sum of $\mathscr{H}_{n}, n \geqslant 0$. Every vector $f \in \mathscr{H}$ is a sequence $f=\left\{f^{(n)}\right\}_{n=0}^{\infty}$ of vectors $f^{(n)} \in \mathscr{H}$ with

$$
\|f\|^{2}=\sum_{n=0}^{\infty}\left\|f^{(n)}\right\|^{2}<\infty
$$

We identify $f^{(n)}$ with the vector $\left\{0,0, \ldots, 0, f^{(n)}, 0, \ldots,\right\} \in \mathscr{H}$ [the $(n+1)$ th component is $f^{(n)}$ and all the other components are zero]. We introduce the subspace

$$
\mathscr{D}_{0}=\left\{f \in \mathscr{P} \mid f^{(n)}=0 \text { for all but finitely many } n\right\},
$$

which is dense in $\mathscr{H}$, and the degree operator ("number operator") $\hat{N}$ by

$$
(\hat{N} f)^{(n)}=n f^{(n)}, \quad n \geqslant 0,
$$

with domain

$$
D(\hat{N})=\left\{f \in \mathscr{H} \mid \sum_{n=0}^{\infty} n^{2}\left\|f^{(n)}\right\|^{2}<\infty\right\} .
$$

The operator $\hat{N}$ is self-adjoint and non-negative.

Let $A$ be a self-adjoint operator in $\mathscr{H}$ which is reduced by each $\mathscr{H}_{n}$, so that for all $n \geqslant 0$,

$$
A: D(A) \cap \mathscr{H}_{n} \rightarrow \mathscr{H}_{n} \text {. }
$$

is self-adjoint. It is easy to see that $A$ is essentially self-adjoint on the dense subspace

$$
\mathscr{D}=D(A) \cap \mathscr{D}_{0} \text {. }
$$

Let $B$ be a symmetric operator in $\mathscr{H}$ that satisfies the following conditions (B1) and (B2).

(B1) $\mathscr{D}_{0} \subset D(B)$ and there exist a constant $c>0$ and a linear operator $L$ in $\mathscr{H}$ such that $D(L) \supset$ $D\left(\left[(A+B)\lceil\mathscr{D}]^{*}\right)\right.$,

$$
L: D(L) \cap \mathscr{H}_{n} \rightarrow \mathscr{H}_{n},
$$

for all $n \geqslant 0$, and

$$
|(f, B g)| \leqslant c\|L f\|\left\|(\hat{N}+1)^{2} g\right\|, \quad f, g \in \mathscr{D} .
$$

(B2) There exists an integer $p \geqslant 0$ such that for all $f \in \mathscr{D}_{0}$,

$$
\left(f^{(m)}, B f^{(n)}\right)=0 \text { unless }|m-n|=0,1, \ldots, p \text {. }
$$

The following theorem gives a criterion for the essential self-adjointness of the operator $A+B$.

Theorem 2.1: Let $A$ and $B$ be as above. Suppose that $A+B$ is bounded from below. Then $A+B$ is essentially selfadjoint on $\mathscr{D}$.

To prove this theorem, we prepare a lemma, which may be interesting in its own right.

Lemma 2.2: Let $S$ and $T$ be symmetric operators in $\mathscr{H}$ and

$$
R \equiv S+T
$$

is strictly positive on a subspace $D \subset D(S) \cap D(T)$, i.e., for a constant $\lambda>0$,

$$
R \geqslant \lambda \text { on } D \text {. }
$$

Let $\left\{f_{n}\right\}_{n=1}^{\infty} \subset D$ be a sequence satisfying the following conditions (i)-(iv): 
(i) $f_{1} \neq 0$;

(ii) $\left(f_{m}, f_{n}\right)=0$, unless $m=n$;

(iii) $\left(f_{m}, S f_{n}\right)=0$, unless $m=n$;

(iv) $\left(f_{m}, T f_{n}\right)=0$, for $|m-n| \geqslant 2$;

(v) $\left(f_{n-1}, T f_{n}\right)+\left(f_{n}, R f_{n}\right)+\left(f_{n+1}, T f_{n}\right)=0, \quad n \geqslant 1$,

where we set $f_{0}=0$. Then, for all $n \geqslant 1, f_{n} \neq 0$ and

$$
\left(f_{n+1}, T f_{n}\right)<0 \text {. }
$$

Moreover,

$$
\sum_{n=1}^{\infty} \frac{1}{\left|\left(f_{n+1}, T f_{n}\right)\right|}<\infty \text {. }
$$

Proof: Conditions (i) and (ii) imply that $\Sigma_{j=1}^{n} f_{j} \neq 0$ for all $n \geqslant 1$. Therefore (2.6) gives

$$
\left(\sum_{j=1}^{n} f_{j}, R \sum_{j=1}^{n} f_{j}\right)>0
$$

Using conditions (iii) $-(v)$, we can show that the left-hand side of (2.10) equals $-\left(f_{n+1}, T f_{n}\right)$. Hence, $f_{n+1} \neq 0$ and (2.8) follows.

To prove (2.9), we define a set of numbers $\left\{a_{n}\right\}_{n=1}^{\infty}$ by the following recursion relations:

$a_{1}=1$,

$$
\begin{aligned}
& a_{n-1}\left(f_{n-1}, T f_{n}\right)+a_{n}\left(f_{n}, R f_{n}\right)+a_{n+1}\left(f_{n+1}, T f_{n}\right) \\
& =\mu a_{n}\left\|f_{n}\right\|^{2}, \quad n \geqslant 1,
\end{aligned}
$$

where $\mu$ is a constant with $0<\mu<\lambda$ and we set $a_{0}=0$. It is easy to see that for all $n \geqslant 2, a_{n}$ is real. Let

$$
g_{n}=a_{n} f_{n}
$$

and

$$
\widehat{R}=R-\mu,
$$

which is strictly positive. Multiplying (2.12) by $a_{n}$, we have

$$
\left(g_{n-1}, T g_{n}\right)+\left(g_{n}, \hat{R} g_{n}\right)+\left(g_{n+1}, T g_{n}\right)=0 \text {. }
$$

Hence, we can apply the preceding result with $f_{n}, R$, and $S$ replaced by $g_{n}, R$, and $S-\mu$, respectively, to obtain

$$
0>\left(g_{n+1}, T g_{n}\right)=-a_{n+1} a_{n}\left|\left(f_{n+1}, T f_{n}\right)\right| .
$$

Since $a_{1}=1>0$, this inequality implies that for all $n \geqslant 2$, $a_{n}>0$. Multiplying (2.7) [resp. (2.12)] by $a_{n}^{2}$ (resp. $a_{n}$ ) and making the subtraction to eliminate the term $\left(f_{n}, R f_{n}\right)$, we obtain

$$
\begin{aligned}
& \left(a_{n}-a_{n+1}\right)\left|\left(f_{n+1}, T f_{n}\right)\right| \\
& \quad=\mu a_{n}\left\|f_{n}\right\|^{2}+\left(a_{n-1}-a_{n}\right)\left|\left(f_{n-1}, T f_{n}\right)\right|, \quad n \geqslant 1 .
\end{aligned}
$$

Taking $n=1$ in this equation, we see that $a_{1}>a_{2}$. It then turns out that for all $n \geqslant 1, a_{n}>a_{n+1}$. Combining this result with (2.13), we obtain

$$
\frac{1}{\left|\left(f_{n+1}, T f_{n}\right)\right|} \leqslant \frac{a_{n}-a_{n+1}}{\mu\left\|f_{1}\right\|^{2}},
$$

which implies that

$$
\sum_{n=1}^{N} \frac{1}{\left|\left(f_{n+1}, T f_{n}\right)\right|} \leqslant \frac{a_{1}}{\mu\left\|f_{1}\right\|^{2}} .
$$

Thus (2.9) follows.

Proof of Theorem 2.1: Without loss of generality, we can assume that for a constant $\gamma>0$,

$$
A+B \geqslant \gamma
$$

on $\mathscr{D}$. Throughout the proof, we set

$$
C=A+B \text {. }
$$

It is sufficient to prove that $\operatorname{Ker}(C \uparrow \mathscr{D})^{*}=\{0\}$ (e.g., Theorem X.26 in Ref. 13). Let $g \in \operatorname{Ker}(C \nmid \mathscr{D})^{*}$. Then

$$
(g, C f)=0 \text {, }
$$

for all $f \in \mathscr{D}$. By (2.4), we have

$$
\begin{aligned}
\left(g^{(n)}, A f\right)=\left(g, A f^{(n)}\right) & =\left(g,(C-B) f^{(n)}\right) \\
& =-\left(g, B f^{(n)}\right) .
\end{aligned}
$$

Using (B1) and (B2), we obtain

$$
\left|\left(g^{(n)}, A f\right)\right| \leqslant c(n+p+1)^{2}\|L g\|\|f\| .
$$

Hence, the map: $f \rightarrow\left(g^{(n)}, A f\right)$ defines uniquely a continuous linear functional on $\mathscr{H}$. Hence, by the Riesz lemma, there exists a vector $\psi_{n} \in \mathscr{H}$ such that

$$
\left(g^{(n)}, A f\right)=\left(\psi_{n}, f\right), \quad f \in \mathscr{D} .
$$

Since $\mathscr{D}$ is a core of $A$, this equation extends to all $f \in D(A)$, which implies that $g^{(n)} \in D\left(A^{*}\right)=D(A)$. Hence, $g^{(n)} \in \mathscr{D}$.

$$
\begin{aligned}
& \text { If } p=0 \text {, then } B: \mathscr{H}_{n} \rightarrow \mathscr{H}_{n} \text { for all } n \geqslant 0 \text { and hence } \\
& C: \mathscr{D} \cap \mathscr{H}_{n} \rightarrow \mathscr{H}_{n},
\end{aligned}
$$

for all $n \geqslant 0$. Therefore, putting $f=g^{(n)} \in \mathscr{D}$ into (2.14), we have

$$
0=\left(g, C g^{(n)}\right)=\left(g^{(n)}, C g^{(n)}\right) \geqslant \gamma\left\|g^{(n)}\right\|^{2} .
$$

Hence, $g^{(n)}=0$ for all $n \geqslant 0$, i.e., $g=0$.

Let $p \geqslant 1$ and define

$$
h_{n}=\sum_{j=0}^{p-1} g^{(p n+j)} \in \mathscr{D} \text {. }
$$

Putting $f=h_{n}$ into (2.14), we have

$$
\left(g, C h_{n}\right)=0 \text {, }
$$

which implies that

$$
\left(h_{n-1}, B h_{n}\right)+\left(h_{n}, C h_{n}\right)+\left(h_{n+1}, B h_{n}\right)=0, \quad n \geqslant 0,
$$

where we set $h_{-1}=0$. It is easy to see that

$$
\left(h_{m}, h_{n}\right)=0 \quad\left(h_{m}, A h_{n}\right)=0,
$$

unless $m=n$ and

$$
\left(h_{m}, B h_{n}\right)=0 \text {, }
$$

for $|m-n| \geqslant 2$. Suppose that $g \neq 0$. Then, for some $n, h_{n} \neq 0$. Hence, we can define

$$
N=\min \left\{n \geqslant 0 \mid \quad\left\|h_{n}\right\| \neq 0\right\} .
$$

Then we can apply Lemma 2.2 with $f_{n}=h_{N_{i-n-1}}(n \geqslant 1)$, $S=A, T=B$, to obtain

$$
K=\sum_{n=N}^{\infty} \frac{1}{\left|\left(h_{n+1}, B h_{n}\right)\right|}<\infty .
$$

Using the Schwarz inequality, we have 


$$
\begin{aligned}
& \sum_{n=N}^{\infty}\left\{\frac{\left.\left\|L h_{n+1}\right\|\left\|h_{n}\right\|\right\}^{1 / 2}}{\left\|\left(h_{n+1}, B h_{n}\right)\right\|}\right\}^{1 / 2}\left\{\sum_{n=N}^{\infty}\left\|L h_{n+1}\right\|^{2}\right\}^{1 / 4}\left\{\sum_{n=N}^{\infty}\left\|h_{n}\right\|^{2}\right\}^{1 / 4} \\
& \leqslant K^{1 / 2} \\
& \leqslant \sqrt{K p\|L g\|\|g\| .}
\end{aligned}
$$

On the other hand, we have by (B1)

$$
\frac{\left\|L h_{n+1}\right\|\left\|h_{n}\right\|}{\left|\left(h_{n+1}, B h_{n}\right)\right|} \geqslant \frac{1}{c p^{2}(n+1)^{2}},
$$

which implies that the left-hand side of (2.15) diverges. This is a contradiction. Thus $g$ must be zero.

\section{ESSENTIAL SELF-ADJOINTNESS OF A CLASS OF HAMILTONIANS IN NONRELATIVISTIC QUANTUM FIELD THEORY}

In this section we apply Theorem 2.1 to prove the essential self-adjointness of a class of Hamiltonians in nonrelativistic quantum field theory. The Hamiltonians we consider correspond to models of a finite number of nonrelativistic particles interacting with some quantum scalar fields.

For a mathematical generality, we assume that the scalar fields under consideration are over $\mathbb{R}^{d}$ with $d \geqslant 1$. The Hilbert space for state vectors of the particle system is taken to be $L^{2}\left(\mathbb{R}^{N}\right)$. We denote by $q=\left(q_{1}, \ldots, q_{N}\right) \in \mathbb{R}^{N}$ the coordinate variable of $\mathbb{R}^{N}$ and define the "momentum operator" $p$ by

$$
p=\left(p_{1}, \ldots, p_{N}\right),
$$

with

$$
p_{j}=-i \frac{\partial}{\partial q_{j}},
$$

where $i=\sqrt{-1}$ and the partial derivatives are taken in the generalized sense.

The mathematical framework for the quantum scalar fields is given as follows: Let $\mathscr{K}$ be the $M$ direct sum of $L^{2}\left(\mathbb{R}^{d}\right)(M \geqslant 1)$ :

$$
\mathscr{K}=\underbrace{L^{2}\left(\mathbb{R}^{d}\right) \oplus \cdots \oplus L^{2}\left(\mathbb{R}^{d}\right)}_{M \text { times }}
$$

and $S^{n}(\mathscr{K})(n \geqslant 1)$ be the $n$-fold symmetric tensor product of $\mathscr{K}$ :

$$
S^{n}(\mathscr{K})=\otimes_{s}^{n} \mathscr{K}
$$

[we set $S^{0}(\mathscr{K})=\mathbb{C}$ ]. The Hilbert space for the scalar fields is taken to be the symmetric Fock space over $\mathscr{K}$ :

$$
\mathscr{F}_{s}(\mathscr{K})=\oplus_{n=0}^{\infty} S^{n}(\mathscr{K}) .
$$

We denote by $a(F)\left(F \in \mathscr{K}^{\prime}\right)$ the annihilation operator in $\mathscr{F}_{s}(\mathscr{K})$ (antilinear in $F$ ) and by $N_{b}$ the number operator. The mapping: $L^{2}\left(\mathbb{R}^{d}\right) \ni f \rightarrow f_{r}=(0, \ldots, 0, f, 0, \ldots, 0) \in \mathscr{K}$ (the $r$ th component is $f$ and the other components are zero) defines an embedding of $L^{2}\left(\mathbb{R}^{d}\right)$ into $\mathscr{K}$. Let $\mathscr{S}\left(\mathbb{R}^{d}\right)$ be the Schwartz space of rapidly decreasing $C^{\infty}$ functions on $\mathbb{R}^{d}$. Then the mapping: . $\mathscr{P}\left(\mathbb{R}^{d}\right) \ni f \rightarrow a\left(f_{r}\right)$ defines an operatorvalued distribution; we denote its kernel by $a_{r}(k)$ :

$$
a\left(f_{r}\right)=\int a_{r}(k) f(k) d k .
$$

The operator-valued distributions $\left\{a_{r}(k)\right\}_{r=1}^{M}$ satisfy the canonical commutation relations:

$$
\begin{aligned}
& {\left[a_{r}(k), a_{s}(p)^{*}\right]=\delta_{r s} \delta(k-p),} \\
& {\left[a_{r}(k), a_{s}(p)\right]=0, r, s=1, \ldots, M .}
\end{aligned}
$$

Let $\mathscr{F}_{0}(\mathscr{K})$ be the finite particle vector space of $\mathscr{F}_{s}(\mathscr{K})$ : $\mathscr{F}_{0}(\mathscr{K})=\left\{\Psi=\left\{\Psi^{(n)}\right\}_{n=0}^{\infty} \in \mathscr{F}_{s}\left(\mathscr{K}^{n}\right) \mid \Psi^{(n)}\right.$

$=0$ for all but finitely many $n\}$.

For each $W_{m, n} \in L^{2}\left(\mathbb{R}^{d(m+n)}\right)(m+n \geqslant 1, m, n \geqslant 0)$ and $r_{i}, s_{j}=1, \ldots, M$, we can define a unique closed linear operator $W_{m, n}\left(a_{r_{1}}^{*}, \ldots, a_{r_{m}}^{*} ; a_{s_{1}}, \ldots, a_{s_{n}}\right)$ in $\mathscr{F}_{s}(\mathscr{K})$ with

$$
D\left(W_{m, n}\left(a_{r_{1}, \ldots,}^{*} a_{r_{m}}^{*} ; a_{s_{1}}, \ldots, a_{s_{n}}\right)\right) \supset \mathscr{F}_{0}(\mathscr{H})
$$

such that $\mathscr{F}_{0}(\mathscr{K})$ is a core for $W_{m, n}\left(a_{r_{1}}^{*}, \ldots, a_{r_{m}}^{*} ; a_{s_{1}}, \ldots, a_{s_{n}}\right)$ and

$$
\begin{aligned}
W_{m, n}\left(a_{r_{1}}^{*}, \ldots, a_{r_{m}}^{*} ; a_{s_{1}}, \ldots, a_{s_{n}}\right) \\
=\int_{\mathbb{R}^{d+m+n},} W_{m, n}\left(k_{1}, \ldots, k_{m}, \xi_{1}, \ldots, \xi_{n}\right) \\
\quad \times\left(\prod_{i=1}^{m} a_{r_{i}}\left(k_{i}\right)^{*}\right)\left(\prod_{j=1}^{n} a_{s_{j}}\left(\xi_{j}\right)\right) d k d \xi
\end{aligned}
$$

as a quadratic form on $\mathscr{F}_{0}(\mathscr{K}) \times \mathscr{F}_{0}(\mathscr{K})$ (see Theorem $X .44$ in Ref. 13). Some fundamental properties of the operator $W_{m, n}\left(a_{r_{3}}^{*}, \ldots, a_{r_{m}}^{*} ; a_{s_{1}}, \ldots, a_{s_{n}}\right)$ are summarized in the following lemma.

Lemma 3.1: (i) If $k$ and $l$ are non-negative integers such that $k+l=m+n$, then

$$
\left(1+N_{b}\right)^{-k / 2} W_{m, n}\left(a_{p_{1}}^{*}, \ldots, a_{r_{m}}^{*} ; a_{s_{1}}, \ldots, a_{s_{n}}\right)\left(1+N_{b}\right)^{-1 / 2}
$$

is a bounded operator with

$$
\begin{aligned}
& \left\|\left(1+N_{b}\right)^{-k / 2} W_{m, n}\left(a_{r_{1}}^{*}, \ldots, a_{r_{m}}^{*} ; a_{s_{1}}, \ldots, a_{s_{n}}\right)\left(1+N_{b}\right)^{-1 / 2}\right\| \\
& \leqslant C(k, l)\|W\|_{L^{2}},
\end{aligned}
$$

where $C(k, l)>0$ is a constant.

$$
\begin{aligned}
& \text { (ii) Let } \\
& \tilde{W}_{m, n}\left(k_{1}, \ldots, k_{m}, \xi_{1}, \ldots, \xi_{n}\right)=W_{n, m}\left(\xi_{1}, \ldots, \xi_{n}, k_{1}, \ldots, k_{m}\right)^{*} \text {. }
\end{aligned}
$$

Then

$$
\begin{aligned}
& D\left(\widetilde{W}_{m, n}\left(a_{s_{1}}^{*}, \ldots, a_{s_{m}}^{*} ; a_{r_{1}}, \ldots, a_{r_{n}}\right)\right) \\
& \quad \subset D\left(W_{n, m}\left(a_{r_{t}}^{*}, \ldots, a_{r_{n}}^{*} ; a_{s_{1}}, \ldots, a_{s_{m}}\right)^{*}\right)
\end{aligned}
$$

and

$\widetilde{W}_{m, n}\left(a_{s_{1}}^{*}, \ldots, a_{s_{m}}^{*} ; a_{r_{1}}, \ldots, a_{r_{n}}\right)=W_{n, m}\left(a_{r_{1}}^{*}, \ldots, a_{r_{n}}^{*} ; a_{s_{1}}, \ldots, a_{s_{m}}\right)^{*}$ on $D\left(\widetilde{W}_{m . n}\left(a_{s_{1}}^{*}, \ldots, a_{s_{m,}}^{*} ; a_{r_{1}}, \ldots, a_{r_{n}}\right)\right)$.

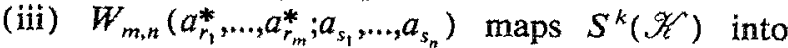
$S^{k+m-n}(\mathscr{K})($ resp. $\{0\})$ for $k \geqslant n$ (resp. $k<n$ ).

For proof of this lemma, see Theorem X.44 in Ref. 13.

Let $\omega_{r}(k), r=1, \ldots, M$, be non-negative measurable functions on $L^{2}\left(\mathbb{R}^{d}\right)$ with $\omega_{r} \in L_{\text {loc }}^{2}\left(\mathbb{R}^{d}\right)$ and

$$
\widehat{\omega}=\oplus_{r=1}^{M} \omega_{r}
$$

be the direct sum of $\omega_{r}$ as multiplication operators. We define

$$
H_{F}=d \Gamma(\hat{\omega})
$$


to be the second quantization of the operator $\hat{\omega}$. We have

$$
H_{F}=\sum_{r=1}^{M} \int \omega_{r}(k) a_{r}(k) * a_{r}(k) d k
$$

as a quadratic form on

$$
\left[\mathscr{F}_{0}(\mathscr{K}) \cap D\left(H_{F}\right)\right] \times\left[\mathscr{F}_{0}(\mathscr{K}) \cap D\left(H_{F}\right)\right] .
$$

The Hilbert space of the coupled system of the particles and the scalar fields is defined by

$$
\mathscr{F}=L^{2}\left(\mathbb{R}^{N}\right) \otimes \mathscr{F}_{s}(\mathscr{K}) .
$$

Every closed operator $A$ (resp. $B$ ) in $L^{2}\left(\mathbb{R}^{N}\right)$ [resp. $\left.\mathscr{F}_{s}(\mathscr{K})\right]$ extends to $\mathscr{F}$ as $A \otimes I$ (rcsp. $I \otimes B$ ), where $I$ denotes identity. In what follows, however, we shall denote them by the same symbols, provided that there is no danger of confusion.

We now consider the following Hamiltonian:

$$
\begin{aligned}
& H=\sum_{j=1}^{N} \frac{p_{j}^{2}}{2 m_{j}}+H_{F}+\sum_{1<k<4}\left\{\lambda_{j_{1} \cdots j_{k}} q_{j_{1}} \cdots q_{j_{k}}+\mu_{j_{1} \cdots j_{k}} p_{j_{1}} \cdots p_{j_{k}}\right\} \\
& +\sum_{1<k<4} \sum_{1<\{<k}\left\{y_{j_{1} \cdots j_{k}} p_{j_{1}} \cdots p_{j_{l}} q_{j_{l+1}} \cdots q_{j_{k}}+v_{j_{1} \cdots j_{k}}^{*} q_{j_{l+1}} \cdots q_{j_{k}} p_{j_{1}} \cdots p_{j_{l}}\right\}+\sum_{1<n+m<4}\left\{W_{m, n}^{\left(r_{1}, \ldots, r_{m}, s_{1}, \ldots, s_{n}\right)}\left(a_{r_{1}}^{*}, \ldots, a_{r_{m}}^{*} ; a_{s_{1}}, \ldots, a_{s_{n}}\right)\right. \\
& \left.+W_{m, n}^{\left(r_{1}, \ldots, r_{m}, s_{1}, \ldots, s_{n}\right)}\left(a_{r_{1}}^{*}, \ldots, a_{r_{m}}^{*} ; a_{s_{1}}, \ldots, a_{s_{n}}\right) *\right\}+\sum_{1<k+m+n<4} \sum_{1<k<k}\left\{\gamma_{j_{1} \cdots j_{k}} p_{j_{1}} \cdots p_{j_{i}} q_{j_{l+1}} \cdots q_{j_{k}}\right. \\
& \left.\times V_{m, n}^{\left(r_{1}, \ldots, r_{m}, s_{1}, \ldots, s_{n}\right)}\left(a_{r_{1}}^{*}, \ldots, a_{r_{m}}^{*} ; a_{s_{1}}, \ldots, a_{s_{n}}\right)+\gamma_{j_{1}}^{*} \ldots j_{k} q_{j_{l+1}} \cdots q_{j_{k}} p_{j_{1}} \cdots p_{j_{i}} V_{m, n}^{\left(r_{1}, \ldots, r_{m}, s_{1}, \ldots, s_{n}\right)}\left(a_{r_{1}}^{*}, \ldots, a_{r_{m}}^{*} ; a_{s_{1}}, \ldots, a_{s_{n}}\right) *\right\},
\end{aligned}
$$

where $m_{j}>0, \lambda_{j_{1} \cdots j_{k}}, \mu_{j_{1} \cdots j_{k}} \in \mathbb{R}, v_{j_{1} \cdots j_{k}}, \gamma_{j_{1} \cdots j_{k}} \in \mathbb{C}$, are constants,

$$
W_{m, n}^{\left(r_{1}, \ldots, s_{n}\right)}, \quad V_{m, n}^{\left(r_{1}, \ldots, s_{n}\right)} \in L^{2}\left(\mathbb{R}^{d(m+n)}\right),
$$

and summations with respect to the repeated indices $j_{l}, r_{i}, s_{i}$, are understood. The operator $H$ is a sum of operators of polynomial type with degree less than or equal to 4 in $q_{j}, p_{j}, a_{r}^{\#}, j=1, \ldots, N, r=1, \ldots, M$. By Lemma 3.1 (i) and the fact that $p_{j}$ and $q_{j}$ leave $\mathscr{S}\left(\mathbb{R}^{N}\right)$ invariant, $H$ is a symmetric operator with

$$
D(H) \supset \mathscr{P}\left(\mathbb{R}^{N}\right) \hat{\otimes}\left(\mathscr{F}_{0}(\mathscr{K}) \cap D\left(H_{F}\right)\right) \equiv \mathscr{D}_{H},
$$

where $\hat{\otimes}$ denotes algebraic tensor product. We prove the following theorem.

Theorem 3.2: Suppose that $H$ is bounded from below on $\mathscr{D}_{H}$. Then $H$ is essentially self-adjoint on $\mathscr{D}_{H}$.

To prove Theorem 3.2 , we need a preliminary. To apply Theorem 2.1 to the present case, we must rewrite $\mathscr{F}$ as an infinite direct sum. To this end, we make use of the FockHermite-Wiener decomposition of $L^{2}\left(\mathbb{R}^{N}\right)$. We first recall this decomposition. Let $v_{j}>0, j=1, \ldots, N$, be constants and introduce the annihilation and creation operators for the particles as follows:

$$
\begin{aligned}
& b_{j}=\left(1 / \sqrt{2 v_{j}}\right)\left(i p_{j}+v_{j} q_{j}\right), \\
& b_{j}^{\dagger}=\left(1 / \sqrt{2 v_{j}}\right)\left(-i p_{j}+v_{j} q_{j}\right), \quad j=1, \ldots, N,
\end{aligned}
$$

which leave $\mathscr{S}\left(\mathbb{R}^{N}\right)$ invariant and satisfy the commutation relations

$$
\left[b_{j}, b_{k}^{\dagger}\right]=\delta_{j k}, \quad\left[b_{j}, b_{k}\right]=0, j, k=1, \ldots, N,
$$

on $\mathscr{S}\left(\mathbf{R}^{N}\right)$. We have from (3.12) and (3.13)

$$
\begin{aligned}
& q_{j}=\left(1 / \sqrt{2 v_{j}}\right)\left(b_{j}+b_{j}^{\dagger}\right), \\
& p_{j}=i \sqrt{v_{j} / 2}\left(b_{j}^{\dagger}-b_{j}\right) .
\end{aligned}
$$

Let

$$
\psi_{0}=\prod_{j=1}^{N}\left\{\left(\frac{v_{j}}{\pi}\right)^{1 / 4} e^{-v \rho_{j}^{2} / 2}\right\} .
$$

Then we have $\left\|\psi_{0}\right\|=1$ and

$$
b_{j} \psi_{0}=0, \quad j=1, \ldots, N
$$

For $z=\left(z_{1}, \ldots, z_{N}\right) \in \mathbb{C}^{N}$, we define the operators $b(z)$ and $b(z)^{\dagger}$ by

$$
b(z)=\sum_{j=1}^{N} z_{j}^{*} b_{j}, \quad b(z)^{\dagger}=\sum_{j=1}^{N} z_{j} b_{j}^{\dagger} .
$$

Then (3.14) is equivalent to the following commutation relations:

$$
\left[b(z), b(w)^{\dagger}\right]=(z, w)_{\mathbf{C}^{N}}, \quad[b(z), b(w)]=0, z, w \in \mathbb{C}^{N} .
$$

Let

$$
\begin{aligned}
\mathscr{M}_{0}= & \left\{\alpha \psi_{0} \mid \alpha \in \mathbb{C}\right\}, \\
\mathscr{M}_{n}= & \left\{\sum_{j_{1}, \ldots, j_{n}=1}^{J_{1}, \ldots, J_{n}} \alpha_{j_{1} \cdots j_{n}} b\left(z_{j_{1}}\right)^{\dagger} \cdots b\left(z_{j_{n}}\right)^{\dagger} \psi_{0} \mid \alpha_{j_{1}} \cdots j_{n}\right. \\
& \left.\in \mathbb{C}, z_{j_{k}} \in \mathbb{C}^{N}, J_{k} \geqslant 1\right\}, \quad n \geqslant 1 .
\end{aligned}
$$

Then one can easily see that

$\mathscr{C}_{n}-\left\{P_{n}(q) \psi_{0} \mid P_{n}\right.$ :polynomials of order $\left.n\right\} \subset \mathscr{S}\left(\mathbb{R}^{N}\right)$.

Hence, for all $n \geqslant 0, \mathscr{M}_{n}$ is finite dimensional. Moreover, we can show that $\mathscr{M}_{n} \perp \mathscr{M}_{m}$ for $m \neq n$ and

$$
L^{2}\left(\mathbb{R}^{N}\right)=\underset{n=0}{\oplus} \mathscr{M}_{n} \cong \mathscr{F}_{s}\left(\mathbb{C}^{N}\right) \text {. }
$$

This is the desired decomposition of $L^{2}\left(\mathbb{R}^{N}\right)$. In the decomposition (3.23), the degree operator is given by

$$
N_{p}=\sum_{j=1}^{N} b_{j}^{\dagger} b_{j} \text {. }
$$

Using (3.23), the entire Hilbert space $\mathscr{F}$ is decomposed as

$$
\mathscr{F}=\underset{n=0}{\oplus} \mathscr{F}_{n},
$$

where 


$$
\mathscr{F}_{n}=\underset{\substack{t+m=n \\ \oplus}}{\oplus} \mathscr{M}_{l} \otimes S^{m}(\mathscr{K})
$$

One can easily show that

$$
\left\|b_{j}^{\#} \psi\right\| \leqslant\left\|\left(N_{p}+1\right)^{1 / 2} \psi\right\|, \quad \psi \in D\left(N_{p}^{1 / 2}\right), j=1, \ldots, N .
$$

Using (3.15), (3.16), and (3.27), we can prove the following estimate:

$$
\begin{aligned}
& \left\|p_{j_{1}} \cdots p_{j_{m}} q_{i_{1}} \cdots q_{i_{n}} \psi\right\| \leqslant C_{m, n}\left\|\left(N_{p}+1\right)^{(m+n) / 2} \psi\right\|, \\
& \psi \in D\left(N_{p}^{(m+n) / 2}\right)
\end{aligned}
$$

where $C_{m, n}>0$ is a constant.

We are now ready to prove Theorem 3.2 .

Proof of Theorem 3.2: Write $H$ as

$$
H=H_{0}+H_{l},
$$

with

$$
H_{0}=\sum_{j=1}^{N} \frac{1}{2 m_{j}}\left(p_{j}^{2}+v_{j}^{2} q_{j}^{2}\right)+H_{F}
$$

and

$$
\begin{aligned}
& H_{I}=-\sum_{j=1}^{N} \frac{\gamma_{j}^{2}}{2 m_{j}} q_{j}^{2}+\sum_{1 \leqslant k \leqslant 4}\left\{\lambda_{j_{1} \cdots j_{h}} q_{j_{i}} \cdots q_{j_{k}}+\mu_{j_{1} \cdots j_{h}} p_{j_{h}} \cdots p_{j_{k}}\right\}
\end{aligned}
$$

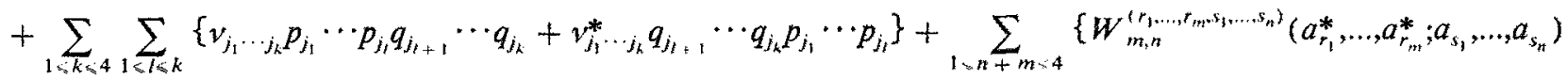

$$
\begin{aligned}
& \left.+W_{m . n}^{\left(r_{1}, \ldots, r_{m, *}, \cdots, s_{n}\right)}\left(a_{r_{1}}^{*}, \ldots, a_{r_{m}}^{*} ; a_{s_{1}}, \ldots, a_{s_{n}}\right)^{*}\right\}+\sum_{1 \leqslant k+\pi+m \leqslant 4} \sum_{1 \leqslant i \leqslant k}\left\{\gamma_{j_{1} \cdots j_{k}} p_{j_{1}} \cdots p_{j}, q_{j_{1}, 1} \cdots q_{j_{k}}\right.
\end{aligned}
$$

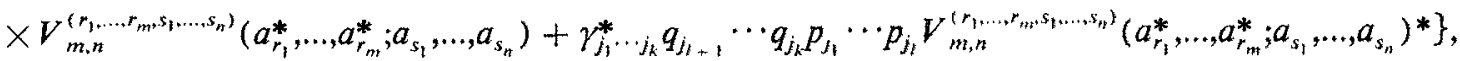

The operator $H_{0}$ is self-adjoint and positive with

$$
D\left(H_{0}\right)=\cap_{j=1}^{N}\left\{D\left(p_{j}^{2}\right) \cap D\left(q_{j}^{2}\right)\right\} \cap D\left(H_{F}\right) .
$$

Since $H_{0}$ is written as

$$
H_{0}=\sum_{j=1}^{N}\left\{\frac{v_{j}}{m_{j}} b_{j}^{\dagger} b_{j}+\frac{v_{j}}{2 m_{j}}\right\}+H_{F},
$$

it follows that $H_{0}$ is reduced by each $\mathscr{F}_{n}$. Let

$$
\mathscr{Q}_{n}=\left\{\Psi=\left\{\Psi^{(n)}\right\}_{n=0}^{\infty} \in \mathscr{F} \mid \Psi^{(n)} \in \mathscr{F}_{n},\right.
$$

and

$$
\left.\Psi^{(n)}=0 \text { for all but finitely many } n\right\}
$$

$$
\mathscr{D}=\mathscr{D}_{0} \cap D\left(H_{0}\right) \text {. }
$$

By (3.22), we can show that

$\mathscr{D}=\left\{P(q) \psi_{\mathrm{o}} \mid P\right.$ :polynomials $\}$

$$
\hat{\otimes}\left[D\left(H_{F}\right) \cap \mathscr{F}_{0}(\mathscr{Y})\right] \subset \mathscr{D}_{H} \text {. }
$$

The degree operator in $\mathscr{F}$ represented as (3.25) is given by

$$
\hat{N}=N_{p}+N_{b} \text {. }
$$

By Lemma 3.1 (iii) and the fact that $b_{j}^{\dagger}$ (resp. $b_{j}$ ) maps $\mathscr{M}_{k}$ into $\mathscr{M}_{k+1}$ (resp. $\mathscr{M}_{k-1}$ ), we see that for all $\Psi=\left\{\Psi^{(n)}\right\}_{n=0}^{\infty}, \Phi=\left\{\Phi^{(n)}\right\}_{n=0}^{\infty} \in \mathscr{D}_{0}$,

$$
\left(\Psi^{(n)}, H_{I} \Phi^{(m)}\right)=0, \quad|m-n|>4 .
$$

Moreover, by Lemma 3.1(i) and (3.28), we can show that

$$
\left\|H_{I} \Psi\right\| \leqslant \sum_{k+l<z} C_{k, l}\left\|\left(N_{p}+1\right)^{k}\left(N_{b}+1\right)^{l} \Psi\right\|, \quad \Psi \in \mathscr{D}_{0},
$$

where $C_{k, l}>0$ is a constant. It is easy to see that

$$
\begin{aligned}
& \left\|\left(N_{p}+1\right)^{k}\left(N_{b}+1\right)^{l} \Psi\right\| \leqslant\left\|(\hat{N}+2)^{k+l} \Psi\right\| \\
& \leqslant 2^{k+l}\left\|(\hat{N}+1)^{k+l} \Psi\right\| .
\end{aligned}
$$

Hence, we get

$$
\left\|H_{I} \Psi\right\| \leqslant C\left\|(\hat{N}+1)^{2} \Psi\right\|,
$$

with $C>0$ being a constant. Thus we can apply Theorem 2.1 with $\mathscr{H}=\mathscr{F}, A=H_{0}, B=H_{1}$ to conclude that $H$ is essentially self-adjoint on $\mathscr{D}$, which, together with (3.29), gives Theorem 3.2.

\section{EXAMPLES}

In this section we discuss some concrete examples of the Hamiltonian $H$ given by (3.10). We follow the notations in Sec. III unless otherwise stated.

Example 1: Let us take $\mathscr{K}=L^{2}\left(\mathbb{R}^{d}\right)$ (i.e., the case $M=1)$, so that

$$
\mathscr{F}=L^{2}\left(\mathbb{R}^{N}\right) \otimes \mathscr{F}_{s}\left(L^{2}\left(\mathbb{R}^{d}\right)\right) .
$$

Let $\omega_{1}=\omega$ and consider the Hamiltonian

$$
\begin{aligned}
H_{1}= & p^{2} / 2 m+V(q)+d \Gamma(\omega) \\
& +\sum_{j=1}^{N} q_{j} \int\left(\lambda_{j}(k) a(k)^{*}+\lambda_{j}(k)^{*} a(k)\right) d k \\
& +\int \frac{\left|\Sigma_{j=1}^{N} \lambda_{j}(k) q_{j}\right|^{2}}{\omega(k)} d k .
\end{aligned}
$$

Here, $m>0$ is a constant, $V(q)$ is a polynomial of the form

$$
V(q)=\sum_{1 \leqslant k<4} g_{j_{1} \cdots j_{k}} g_{j_{1}} \cdots q_{j_{k}},
$$

with $g_{j, \cdots j_{k}} \in \mathbb{R}$ and $\lambda_{j}$ is a measurable function on $\mathbb{R}^{d}$ with $\lambda_{j}, \lambda_{j} / \sqrt{\omega} \in L^{2}\left(\mathbb{R}^{d}\right)$. We assume that $V$ is bounded from below:

$$
\inf _{q \in \mathbb{R}^{d}} V(q)>-\infty .
$$

In the case where $N=1$ and $V(q)=K q^{2} / 2$ with a constant $K>0, H_{1}$ gives the Hamiltonian of a model of laser, ${ }^{2}$ which was discussed rigorously in Refs. 4 and 5 , in connection with the problem of Lamb shift and spontaneous emission of light in quantum electrodynamics. The case where $N=d=3$ and 
$V(q)=K q^{2} / 2$ is the linear polaron model. ${ }^{3}$ The Hamiltonian $H_{1}$ with $N=1$ and with a nonquadratic $V$ was proposed by Caldeira and Leggett ${ }^{11}$ to discuss quantum tunneling and coherence with dissipation. For quantum coherence, $V$ is taken to be a double well potential, e.g., $V(q)=g\left(1-q^{2}\right)^{2}$ with a constant $g>0$. The model given by $(4.1)$ is a generalization of these models.

$$
\mathscr{D}_{H_{1}}=\mathscr{S}\left(\mathbb{R}^{N}\right) \hat{\otimes}\left[\mathscr{F}_{0}\left(L^{2}\left(\mathbb{R}^{d}\right)\right) \cap D(d \Gamma(\omega))\right] \text {. }
$$

Theorem 4.1: The operator $H_{1}$ is bounded from below and essentially self-adjoint on $\mathscr{D}_{H_{1}}$.

Proof: Since $H_{1}$ is of the form of the operator $H$ defined by (3.10), we need only to show that it is bounded from below. Then Theorem 3.2 gives the essential self-adjointness of $H_{1}$ on $\mathscr{T}_{H_{1}}$. Introducing the operators

$$
\begin{aligned}
H_{b}= & d \Gamma(\omega)+\sum_{j=1}^{N} q_{j} \int\left(\lambda_{j}(k) a(k)^{*}+\lambda_{j}(k)^{*} a(k)\right) d k \\
& +\int \frac{\left|\Sigma_{j=1}^{N} \lambda_{j}(k) q_{j}\right|^{2}}{\omega(k)} d k
\end{aligned}
$$

and

$$
H_{p}=p^{2} / 2 m+V(q),
$$

we can write $H_{1}$ as

$$
H_{1}=H_{p}+H_{b} \text {. }
$$

By (4.3), $H_{p}$ is bounded from below. We note that for all $\Psi \in \mathscr{D}_{H_{1}}$,

$$
\begin{aligned}
\left(\Psi, H_{b} \Psi\right)= & \int\left(\left(\sqrt{\omega(k)} a(k)+\sum_{j=1}^{N} \frac{\lambda_{j}(k) q_{j}}{\sqrt{\omega(k)}}\right) \Psi,\right. \\
& \left.\left(\sqrt{\omega(k)} a(k)+\sum_{j=1}^{N} \frac{\lambda_{j}(k) q_{j}}{\sqrt{\omega(k)}}\right) \Psi\right) d k .
\end{aligned}
$$

The right-hand side is non-negative and hence $\left(\Psi, H_{b} \Psi\right) \geqslant 0$. Thus it follows that $H_{1}$ is bounded from below on $\mathscr{D}_{H_{1}}$.

Example 2: Let $b_{j}^{\#}$ be given by (3.12) and (3.13) and $V$ be as in Example 1. Let

$$
\begin{aligned}
H_{2}= & p^{2} / 2 m+V(q)+d \Gamma(\omega) \\
& +\sum_{j=1}^{N} \int\left(\lambda_{j}(k) b_{j} a(k)^{*}+\lambda_{j}(k)^{*} b_{j}^{\dagger} a(k)\right) d k \\
& +\sum_{i, j=1}^{N} \int d k \frac{\lambda_{i}(k)^{*} \lambda_{j}(k)}{\omega(k)} b_{i}^{\dagger} b_{j},
\end{aligned}
$$

This model is a variant of Example 1. The case where $N=1$ and

$$
H_{p}=\left(v_{1} / m\right) b_{1}^{\dagger} b_{1}
$$

is called the RWA oscillator (e.g., Refs. 6-8).

Theorem 4.2: The operator $H_{2}$ is bounded from below and essentially self-adjoint on $\mathscr{D}_{H_{1}}$.

Proof: The operator $\mathrm{H}_{2}$ is of the form of $H$ given by (3.10). Hence, we need only to prove the boundedness from below of $H_{2}$ on $\mathscr{D}_{H_{1}}$. This can be done in the same way as in the proof of Theorem 4.1; in fact, we can show that $H_{2}-H_{p} \geqslant 0$ on $\mathscr{P}_{H_{1}}$.

Example 3: We consider the following case:

$$
\begin{aligned}
& N=d, \quad m_{j}=m, \quad j=1, \ldots, d, \\
& \mathscr{K}=\oplus^{d-1} L^{2}\left(\mathbb{R}^{d}\right) \quad(\text { i.e., } M=d-1), \\
& \omega_{r}(k)=\omega(k), \quad r=1, \ldots, d-1,
\end{aligned}
$$

so that

$$
\mathscr{F}=L^{2}\left(\mathbb{R}^{d}\right) \otimes \mathscr{F}_{s}\left(\oplus^{d-1} L^{2}\left(\mathbb{R}^{d}\right)\right) .
$$

This choice gives a framework to discuss models of a $d$-dimensional electron coupled to a quantized radiation field. ${ }^{9,10,14,15}$ Let $\rho(x)$ be a real distribution on $\mathbb{R}^{d}$ such that its Fourier transform $\hat{\rho}(k)$ is a measurable function with

$$
\hat{\rho} / \sqrt{\omega}, \quad|k| \hat{\rho} / \sqrt{\omega} \in L^{2}\left(\mathbb{R}^{d}\right)
$$

and $\left\{e^{(r)}(k)\right\}_{r=1}^{d-1}$ be a set of vectors in $\mathbb{R}^{d}$ ("polarization vectors" of a photon with momentum $k$ ) such that

$$
e^{(r)}(k) \cdot e^{(s)}(k)=\delta_{r s}, \quad k \cdot e^{(r)}(k)=0, r, s=1, \ldots, d-1 .
$$

The time zero radiation field with cutoff $\hat{\rho}$ is defined by

$$
\begin{aligned}
A_{j}(x ; \rho)= & \int \frac{1}{\sqrt{2 \omega(k)}} e_{j}^{(r)}(k)\left\{\hat{\rho}(k) a_{r}(k)^{*}\right. \\
& \left.\times e^{-i k x}+\hat{\rho}(k)^{*} a_{r}(k) e^{i k x}\right\} d k, \quad j=1, \ldots, d .
\end{aligned}
$$

We consider the following Hamiltonian:

$$
\begin{aligned}
H_{3}= & \frac{1}{2 m}\left(p-e A(0 ; \rho)-\lambda e \sum_{j=1}^{d} q_{j}\left(\partial_{j} A\right)(0 ; \rho)\right)^{2} \\
& +V(q)+d \Gamma\left(\oplus^{d-1} \omega\right),
\end{aligned}
$$

where $e \in \mathbb{R}$ is a parameter denoting the elementary charge, $\lambda \in \mathbb{R}$, and $V(q)$ is a polynomial given by (4.2) with (4.3) $(N=d-1)$. The case $\lambda=0$ corresponds to the usual dipole approximation. The case where $V(q)=\epsilon q^{2} / 2(\epsilon>0)$ and $\lambda=0$ has been discussed in Ref. 10 (cf. also Ref. 9 ). Let $\mathscr{D}_{H_{3}}=\mathscr{S}\left(\mathbb{R}^{d}\right) \hat{\otimes}\left[D\left(d \Gamma\left(\oplus^{d-1} \omega\right)\right)\right.$

$$
\left.\cap \mathscr{F}_{\mathrm{o}}\left(\oplus^{d-1} L^{2}\left(\mathbb{R}^{d}\right)\right)\right] \text {. }
$$

Theorem 4.3: The operator $H_{3}$ is bounded from below and essentially self-adjoint on $\mathscr{D}_{H_{3}}$.

Proof: The operator $\mathrm{H}_{3}$ is also of the form of $H$ given by (3.10). It is obvious that $H_{3}$ is bounded from below on $\mathscr{D}_{H_{3}}$. Thus, applying Theorem 3.1, we get the desired result.

Remarks: In the case $\lambda=0$, the second condition for $\hat{\rho}$ in (4.7) can be dropped. The above theorem slightly improves the result in Ref. 10 on the essential self-adjointness of $H_{3}$ with $V(q)=\epsilon q^{2} / 2$ and with $\lambda=0$ in the sense that concerning the condition for $\rho$, we need to assume only the first condition in (4.7), while in Ref. 10, we assume, in addition to the first condition in (4.7), $\sqrt{\omega} \rho \in L^{2}\left(\mathbb{R}^{d}\right)$.

Example 4: Scalar quantum electrodynamics with cutoffs. We consider a quantum system of a charged scalar field interacting with a radiation field. The Fock space to describe such a system is given by

$$
\mathscr{F}=\mathscr{F}_{s}(\mathscr{K})
$$

(i.e., the case " $N=0$ " in the framework given in Sec. III), where $\mathscr{K}$ is given by (3.2) with $M=2+(d-1)$. We set $a_{1}(k)=b(k), \quad a_{2}(k)=c(k), \quad \omega_{1}(k)=\omega_{2}(k)=\mu(k)$,

$$
\omega_{3}(k)=\cdots=\omega_{d+1}(k)=\omega(k),
$$

and rename $a_{r}(k), r=3, \ldots, 2+(d-1)$ as $a_{r}(k), r$ 
$=1, \ldots, d-1$, respectively. Let $\eta$ be a measurable function on $\mathbb{R}^{d}$ such that

$$
\hat{\eta} / \sqrt{\mu}, \quad|k| \hat{\eta} / \sqrt{\mu}, \quad \sqrt{\mu} \hat{\eta} \in L^{2}\left(\mathbb{R}^{d}\right) .
$$

Then the time-zero charged scalar field and its conjugate with the ultraviolet cutoff $\hat{\eta}$ are defined by

$$
\begin{aligned}
\phi(x ; \eta)= & \int \frac{1}{\sqrt{2 \mu(p)}}\left\{\hat{\eta}(p) c(p)^{*} e^{-i p x}\right. \\
& \left.+\hat{\eta}(p)^{*} b(p) e^{i k p}\right\} d p, \\
\pi(x ; \eta)= & i \int \sqrt{\frac{\mu(p)}{2}}\left\{\hat{\eta}(p) b(p)^{*} e^{-i p x}\right. \\
& \left.-\hat{\eta}(p)^{*} c(p) e^{i k p}\right\} d p .
\end{aligned}
$$

We consider the following Hamiltonian:

$$
\begin{aligned}
H_{4}= & \int g(x)\left\{\pi(x ; \eta)^{*} \pi(x ; \eta)\right. \\
& +(\nabla+i e A(x ; \rho)) \phi(x ; \eta) *(\nabla-i e A(x ; \rho)) \phi(x ; \eta) \\
& \left.+m^{2} \phi(x ; \eta)^{*} \phi(x ; \eta)\right\} d x+H_{E M},
\end{aligned}
$$

where

$$
H_{E M}=\sum_{r=1}^{d-1} \int \omega(k) a_{r}(k) * a_{r}(k) d k
$$

and $g \in \mathscr{P}\left(\mathbb{R}^{d}\right)$ is positive.

Theorem 4.4: The operator $H_{4}$ is non-negative and essentially self-adjoint on $D\left(H_{E M}\right) \cap \mathscr{F}_{0}\left(\mathscr{F}^{\prime}\right)$.
Proof: One can easily check that $H_{4}$ is of the form of $H$ given by (3.10) and is non-negative on $D\left(H_{E M}\right) \cap \mathscr{F}_{0}\left(\mathscr{K}^{\prime}\right)$. Thus we can apply Theorem 3.1 to obtain the desired result.

For formal aspects of the model $\mathrm{H}_{4}$ without cutoffs, see, e.g., Ref. 12.

\section{ACKNOWLEDGMENT}

This work was supported by the Grant-In-Aid 02740094 for science research from the Ministry of Education, Japan.

'D. Masson and W. K. McClary, Commun. Math. Phys. 21, 71 (1971).

${ }^{2}$ F. Schwabl and W. Thirring, Ergb. Exakt. Naturwiss. 36, 219 (1964),

${ }^{3}$ N. N. Bogoliubov and N. N. Bogoliubov, Jr., Some Aspects of Polaron Theory (World Scientific, Sigapore, 1988).

${ }^{4}$ A. Arai, J. Math. Phys. 22, 2539 (1981).

${ }^{5}$ A. Arai, J. Math. Phys. 22, 2549 (1981).

${ }^{6}$ B. Braun, Physica A 129, 262 (1985).

${ }^{7}$ K. Lindenberg and B. J. West, Phys. Rev. A. 30, 568 (1984).

${ }^{8}$ A. Arai, J. Math. Anal. Appl. 140, 270 (1989).

${ }^{9}$ A. Arai, J. Math. Phys. 24, 1896 (1983).

${ }^{10}$ A. Arai, J, Math. Phys. 32, 2224 (1991).

"A. O. Caldeira and A. J. Leggett, Ann. Phys. (NY) 149, 374 (1983).

${ }^{12}$ C. Itzykson and J. B. Zuber, Quantum Field Theory (McGraw-Hill, New York, 1980).

${ }^{13}$ M. Reed and B. Simon, Methods of Modern Mathematical Physics II: Fourier Analysis, Self-adjointness (Academic, New York, 1975).

${ }^{14}$ A. Arai, J. Math. Phys. 22, 534 (1981).

${ }^{15}$ A. Arai, J. Math. Phys. 31, 2653 (1990). 
Journal of Mathematical Physics is copyrighted by the American Institute of Physics (AIP). Redistribution of journal material is subject to the AIP online journal license and/or AIP copyright. For more information, see http://ojps.aip.org/jmp/jmpcr.jsp Copyright of Journal of Mathematical Physics is the property of American Institute of Physics and its content may not be copied or emailed to multiple sites or posted to a listserv without the copyright holder's express written permission. However, users may print, download, or email articles for individual use. 